\title{
Compression limits in cascaded quadratic soliton compression
}

\author{
Bache, Morten; Bang, Ole; Krolikowski, Wieslaw; Moses, Jeffrey; Wise, Frank W.
}

\section{Published in:}

Opto-Electronics and Communications Conference, 2008 and the 2008 Australian Conference on Optical Fibre Technology. OECC/ACOFT 2008. Joint conference of the

Link to article, DOI:

10.1109/OECCACOFT.2008.4610499

Publication date:

2008

Document Version

Publisher's PDF, also known as Version of record

Link back to DTU Orbit

\section{Citation (APA):}

Bache, M., Bang, O., Krolikowski, W., Moses, J., \& Wise, F. W. (2008). Compression limits in cascaded quadratic soliton compression. In Opto-Electronics and Communications Conference, 2008 and the 2008 Australian Conference on Optical Fibre Technology. OECC/ACOFT 2008. Joint conference of the (pp. 1-2). IEEE. https://doi.org/10.1109/OECCACOFT.2008.4610499

\section{General rights}

Copyright and moral rights for the publications made accessible in the public portal are retained by the authors and/or other copyright owners and it is a condition of accessing publications that users recognise and abide by the legal requirements associated with these rights.

- Users may download and print one copy of any publication from the public portal for the purpose of private study or research.

- You may not further distribute the material or use it for any profit-making activity or commercial gain

- You may freely distribute the URL identifying the publication in the public portal 


\title{
Compression limits in cascaded quadratic soliton compressors
}

\author{
Morten Bache, ${ }^{1}$ Ole Bang, ${ }^{1}$ Wieslaw Krolikowski, ${ }^{2}$ Jeff Moses,${ }^{3}$ and Frank W. Wise ${ }^{4}$ \\ ${ }^{1}$ DTU Fotonik, Dept. of Photonics Engineering, Technical University of Denmark, Bld. 343, DK-2800 Lyngby, Denmark \\ ${ }^{2}$ Research School of Physical Sciences and Engineering, Australian National University, Canberra ACT 0200, Australia \\ ${ }^{3}$ Optics and Quantum Electronics Group, Massachusetts Institute of Technology, Cambridge, MA 02139, USA \\ ${ }^{4}$ Dept. of Applied and Engineering Physics, Cornell University, Ithaca, New York 14853, USA \\ Phone: +45 45256373, Fax: +45 45936581, E-mail: bang@com.dtu.dk
}

\begin{abstract}
Cascaded quadratic soliton compressors generate under optimal conditions few-cycle pulses. Using theory and numerical simulations in a nonlinear crystal suitable for high-energy pulse compression, we address the limits to the compression quality and efficiency.
\end{abstract}

\section{Introduction}

Soliton compressors are attractive because only a single nonlinear medium is needed to achieve many-fold pulse compression. In cascaded quadratic soliton compressors (CQSCs) soliton compression of high-energy fs pulses is possible, and few-cycle pulses can be reached in the near-infrared [1-6]. The cascaded quadratic nonlinearity is achieved by phase-mismatched second-harmonic generation (SHG), where the fundamental wave (FW) experiences a strong nonlinear phase shift from the cyclic energy transfer to the second harmonic $(\mathrm{SH})$. Unique for the CQSC is that solitons exist with normal dispersion, because the effective cubic nonlinearity induced by the cascaded SHG can be made selfdefocusing [1]. Consequently the CQSC can compress arbitrarily high-energy pulses, and soliton compression may occur even in the near-infrared where the absence of anomalous dispersion prevents traditional soliton compressors to work. It is well known that in the stationary regime clean compression is possible in the the CQSC, while in the nonstationary regime groupvelocity mismatch (GVM, given by the parameter $\left.d_{12}\right)$ distorts the compressed pulse $[1,3]$. Using nonlocal theory we showed that the GVM-induced Raman-like term found previously [3] originates from a temporally nonlocal response function [4], and an accurate transition to the stationary regime was found. Here we seek to investigate in details what really limits the compression [6].

\section{Nonlocal theory}

The CQSC works with the phase-mismatch $\Delta k=k_{2}-$ $2 k_{1} \gg 0$ to get a self-defocusing cascaded nonlinearity, so solitons exist with normal FW group-velocity disper$\operatorname{sion}(\mathrm{GVD}) k_{1}^{(2)}=\partial^{2} k_{1} /\left.\partial \omega^{2}\right|_{\omega=\omega_{1}}>0$. In the cascading limit $|\Delta k| L_{\mathrm{D}, 1} \gg 1$ the usual coupled SHG equations can be reduced to a single equation for the $\mathrm{FW}$ [4], neglecting HOD, self-steepening and Kerr cross-phase modulation (XPM) effects

$$
\begin{gathered}
{\left[i \partial_{\xi}-\frac{1}{2} \partial_{\tau \tau}\right] U_{1}+N_{\mathrm{Kerr}}^{2} U_{1}\left|U_{1}\right|^{2}} \\
=N_{\mathrm{SHG}}^{2} U_{1}^{*} \int_{-\infty}^{\infty} \mathrm{d} s R_{ \pm}(s) U_{1}^{2}(\xi, \tau-s)=0 .
\end{gathered}
$$

The quadratic (SHG) and cubic (Kerr) soliton numbers are $N_{\mathrm{SHG}}^{2}=L_{\mathrm{D}, 1} \mathcal{E}_{0}^{2} \omega_{1}^{2} d_{\mathrm{eff}}^{2} / c^{2} n_{1} n_{2}|\Delta k|$ and $N_{\mathrm{Kerr}}^{2}=$
$L_{\mathrm{D}, 1} n_{\mathrm{Kerr}, 1} \mathcal{E}_{0}^{2} \omega_{1} / c[3,5]$, where $d_{\text {eff }}$ is the effective quadratic nonlinearity, and $n_{\mathrm{Kerr}, j}$ is the cubic (Kerr) nonlinear refractive index. Time and the propagation coordinate are normalized to the $\mathrm{FW}$ input duration $T_{0}$ and the FW GVD length $L_{\mathrm{D}, 1}=T_{0}^{2} /\left|k_{1}^{(2)}\right|$, and $U_{1}=$ $E_{1} / \mathcal{E}_{0}$ is scaled to the peak input electric field. This generalized nonlinear Schrödinger equation (NLSE) shows that the cascaded quadratic nonlinearity imposes a temporal nonlocal response on the FW, with the nonlocal response functions

$$
\begin{aligned}
& R_{+}(\tau)=\frac{\tau_{a}^{2}+\tau_{b}^{2}}{2 \tau_{a}^{2} \tau_{b}} \exp \left(-i s_{a} \tau / \tau_{a}\right) \exp \left(-|\tau| / \tau_{b}\right) \\
& R_{-}(\tau)=\frac{\tau_{a}^{2}-\tau_{b}^{2}}{2 \tau_{a}^{2} \tau_{b}} \exp \left(-i s_{a} \tau / \tau_{a}\right) \sin \left(|\tau| / \tau_{b}\right)
\end{aligned}
$$

where the dimensionless nonlocal time scales $\tau_{a}$ and $\tau_{b}$ depend on the FW and SH dispersion. According to the nonlocal theory the GVM effects dominate for $\Delta k<d_{12}^{2} / 2 k_{2}^{(2)}$ : this nonstationary regime is controlled by the oscillatory response function $R_{-}$. For $\Delta k>d_{12}^{2} / 2 k_{2}^{(2)}$ the cascaded nonlinearities dominate: this stationary regime is controlled by the localized response function $R_{+}$.

\section{Weakly nonlocal limit}

In the weakly nonlocal limit, where the nonlocal response is much faster than the response of $U_{1}^{2}$, Eq. (1) can be approximated as [6]

$$
\begin{aligned}
& {\left[i \partial_{\xi}-\frac{1}{2} \partial_{\tau \tau}\right] U_{1}-N_{\mathrm{eff}}^{2} U_{1}\left|U_{1}\right|^{2}} \\
& =N_{\mathrm{SHG}}^{2}\left[i s_{a} \tau_{R, \mathrm{SHG}}\left|U_{1}\right|^{2} \partial_{\tau} U_{1}+\frac{1-s_{b}}{2} U_{1}^{*} \rho\left(\tau, U_{1}\right)\right]
\end{aligned}
$$

where $s_{b}=+1\left(s_{b}=-1\right)$ in the stationary (nonstationary) regime, and $s_{a}=\operatorname{sgn}\left(d_{12} k_{2}^{(2)}\right)$. The LHS is an NLSE supporting solitons if the effective soliton order $N_{\text {eff }}=\left(N_{\mathrm{SHG}}^{2}-N_{\mathrm{Kerr}}^{2}\right)^{1 / 2}$ is above unity. $N_{\text {eff }}$ also controls the compressor performance through the NLSE-like scaling laws [5]. The RHS gathers two detrimental terms: (1) A GVM-induced Raman-like perturbation with a characteristic dimensionless time $\tau_{R, \mathrm{SHG}} \equiv 2\left|d_{12} / \Delta k T_{0}\right|$. (2) A GVM-induced term $U_{1}^{*} \rho\left(\tau, U_{1}\right)$ containing oscillatory components with periods dictated by $\tau_{a}$ and $\tau_{b}$, which explains the trailing oscillations often observed in the nonstationary regime, see Fig. 1(a) for $\Delta k=30 \mathrm{~mm}^{-1} . \rho\left(\tau, U_{1}\right)$ is caused by the oscillatory nature of $\left|R_{-}\right|$, so it appears only in the nonstationary regime. The RHS of Eq. (4) holds another insight: for a given $\Delta k$, increasing $N_{\mathrm{SHG}}$ by increasing 
the intensity does not necessarily lead to better compression because the Raman-like term, which causes strong pulse asymmetry and soliton splitting, scales as $N_{\mathrm{SHG}}^{2}$. Similarly in the nonstationary regime, the detrimental oscillatory term $U_{1}^{*} \rho\left(t, U_{1}\right)$ also scales as $N_{\mathrm{SHG}}^{2}$.

\section{Numerical results and discussion}

Fig. 1 shows numerics all having the same soliton or$\operatorname{der} N_{\text {eff }}=8$. Thus, the 200 fs input pulse should in all cases be compressed to $6.0 \mathrm{fs}$ [5]. This is indeed observed in the stationary regime for $\Delta k=50 \mathrm{~mm}^{-1}$. For larger $\Delta k$, still in the stationary regime, Kerr XPM gradually degrades compression. For smaller $\Delta k$ the transition to the nonstationary regime is approached $\left(\Delta k=43 \mathrm{~mm}^{-1}\right)$, where pulse compression is limited by the nonlocal time scale $t_{b}=\tau_{b} T_{0}$. In the nonstationary regime $(\Delta k<42)$, pulse compression degrades due to increasing Raman-like effects $\left(\tau_{R, \mathrm{SHG}} \propto\right.$ $\Delta k^{-1}$ ), and to slow trailing oscillations (evident for $\Delta k=30 \mathrm{~mm}^{-1}$ ), caused by the GVM-induced oscillatory term $\rho$ in Eq. (4). All simulations have a FW peak around $3 \mu \mathrm{m}$, which is a dispersive wave phase-matched to the FW soliton, causing the fast trailing oscillations for $\Delta k=50,43,41 \mathrm{~mm}^{-1}$; these prevent reaching single-cycle pulses for larger $N_{\text {eff }}$. In the nonstationary regime a distinct red-shifted peak appears in the $\mathrm{SH}$ spectrum at a frequency $\Omega_{+}$determined by the nonlocal theory. In turn, close to the transition $\left(\Delta k=41 \mathrm{~mm}^{-1}\right)$ the FW has a corresponding spectral hole at $\Omega_{+}$, while further away $\left(\Delta k=30 \mathrm{~mm}^{-1}\right)$ it becomes a spectral peak. We show in Fig. 1(d) the red-shifted holes/peaks found numerically versus $\Delta k$, with an impressive agreement with the nonlocal theory.

\section{Conclusions}

In summary the compression limits in the nonstationary regime are the GVM-induced Raman-like effects and oscillatory components. In the stationary regime the GVM-induced Raman-like effects, nonlocal effects, competing cubic nonlinearities and XPM effects, and dispersive waves, which only exist when taking into account higher-order dispersion, all limit compression.

\section{References}

[1] X. Liu, L. Qian, and F. W. Wise, Opt. Lett. 24 (1999) p. 1777

[2] S. Ashihara, J. Nishina, T. Shimura, and K. Kuroda, J. Opt. Soc. Am. B 19 (2002) p. 2505

[3] J. Moses and F. W. Wise, Opt. Lett. 31 (2006) p. 1881

[4] M. Bache, O. Bang, J. Moses, and F. W. Wise, Opt. Lett. 32 (2007) p. 2490

[5] M. Bache, J. Moses, and F. W. Wise, J. Opt. Soc. Am. B 24 (2007) p. 2752

[6] M. Bache et al., Opt. Express 16 (2008) p. 3273
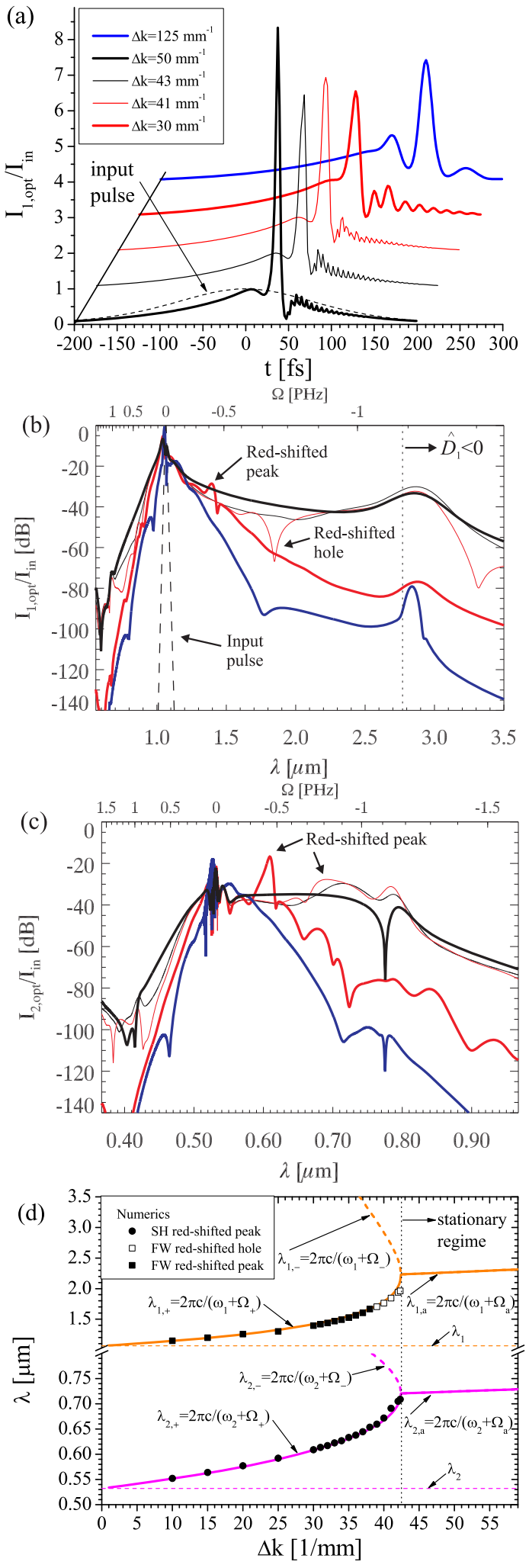

Fig. 1: Soliton compression with $N_{\text {eff }}=8$ of a $200 \mathrm{fs}$ FWHM $\lambda_{1}=1064 \mathrm{~nm}$ pulse in a BBO crystal. (a) FW time plot, (b) the FW and (c) SH spectra at the optimal compression point. (d) The red-shifted spectral peaks in the nonstationary regime from numerics (symbols) and nonlocal theory (lines). The full coupled SHG equations are used, including self-steepening on all nonlinear terms and higher-order dispersion. 